\title{
L'ARCHEOLOGIA SPERIMENTALE COME METODO DI INDAGINE PER LO STUDIO DElle Veneri del Paleolitico Superiore
}

\section{Experimental Archaeology as a Method for Studying the Paleolithic Venus Figurines}

\author{
MARTINA BASILE ${ }^{1}$
}

(1) Università di Roma “La Sapienza”.martina1811@tiscali.it

\section{Riassunto:}

Nel presente articolo si vuole proporre una metodologia di studio delle Veneri del Paleolitico Superiore utilizzando i principi scientifici della Archeologia Sperimentale. L'obiettivo era quello di comprendere come e con quali azioni gli artisti preistorici avevano realizzato le Veneri Paleolitiche. Si è presa come Venere di riferimento la statuina in osso trovata in località Parabita (Lecce, Puglia), chiamata "Venere Grande”, e si è fissato un protocollo sperimentale che prevedeva il confronto tra le tracce tecnologiche presenti sulla superficie della Venere Archeologica e quelle create sperimentalmente. I campioni sperimentali consistevano, nella creazione di una serie di tracce tecnologiche su oggetti in osso realizzati con strumenti litici affini a quelli trovati nel sito di Parabita e, la creazione, di una Venere Campione, simile alla statuina archeologica. Il risultato finale è stata la creazione di un riassunto schematizzato delle azioni effettuate per la realizzazione della Venere Grande.

Parole chiave: Archeologia Sperimentale, Veneri Paleolitiche, Gravettiano Finale/Epigravettiano iniziale, tracce tecnologiche, osso

\section{Abstract:}

In this paper we propose a new methodology to approach the study of the Upper Paleolithic Venus figurines using the scientific principles of experimental archaeology. The main aim is to understand the chaine operatoire and how the prehistoric artists made the Paleolithic Venus figurines. The bone figurine found in Parabita (Lecce, Puglia) known as "Venere Grande" is used as case study. It has been developed an experimental protocol, that implies the comparison between the technological traces present on the Paleolithic Venus figurine surface and the one experimentally recreated.

The experimental samples consists of the realisation of series of technological traces on bone objects with lithics similar to those found at the Parabita site and also the reproduction of a Venus figurine that looks like the original one. The final result has been the schematic description of the actions involved in the making of the "Venere Grande".

Key Words: Experimental Archaeology, Paleolithic figurines, Final Gravettian/Early Epigravettian, technological traces, bone

Copyright: (C) 2016 Martina Basile. This is an open access paper distributed under the terms of the 


\section{INTRODUZIONE}

La presente ricerca ha come obiettivo la messa a punto di una metodologia di studio delle Veneri del Paleolitico Superiore utilizzando i presupposti dell'Archeologia Sperimentale.

L'archeologia sperimentale, nata negli anni sessanta, grazie ai principi scientifici proposti dalla New Archeology (Binford 1968) ha come scopo quello di verificare interpretazioni archeologiche tramite la formulazione di protocolli sperimentali a parametri controllati. In pratica si effettua la riproduzione di oggetti, strutture, situazioni con materiali e tecniche usate nel passato, creando una replica, nel caso in cui l'oggetto archeologico è a nostra disposizione, o una ricostruzione tecnica in caso contrario (Coles 1973). In senso teorico si può dire che attraverso questa metodologia le domande come: cosa facevano gli antichi? Come? Perché? trovano una risposta scientifica attraverso l'azione sperimentale, che può essere una riproduzione di artefatti o simulazioni delle azioni passate (Reynolds 1999).

L'archeologia sperimentale è quindi un metodo di ricerca utile per la ricostruzione degli oggetti e della gestualità del passato. La riproduzione di questi oggetti avviene con l'utilizzo di materiali il più possibile affini all'originale e seguendo un protocollo sperimentale, vale a dire, l'applicazione di una serie di azioni che sono replicabili ovunque e da chiunque in modo che il risultato sia verificabile in qualsiasi contesto. Questa metodologia, fondata su azioni ripetibili e verificabili scientificamente, è stata applicata raramente allo studio delle evidenze artistiche del Paleolitico, benché, abbia delle potenzialità interpretative eccezionali in un campo da sempre soggetto ad analisi estetico-tipologiche. Le sole analisi estetico-tipologiche posso essere alterate dalla soggettività di ciascun individuo in quanto basate su valutazioni indotte dalla materia stessa dell'arte. Si può partire, quindi, dalla definizione di arte L'arte è una attivitàolistica costituita da una attività-teorica fondata sull'intuizione che determina una attività-pratica in cui il valore dell'opera realizzata risulta individuato dal suo significato etico, estetico e spirituale (Sarno 1989). In pratica l'arte consiste di tre momenti fondamentali e inscindibili, ossia "pensare" l'opera (attività teorica), realizzarla concretamente (attività pratica) e infondervi un significato (attività spirituale). L'ultima attività, oltre a essere totalmente suscettibile di soggettività è quindi distinta da cultura a cultura, è unita al concetto di simbolo.
Per simbolo si intende un qualsiasi oggetto, o segno, che rappresenta un'altra cosa in virtù della sua corrispondenza o analogia. (Rodriguez 2000). Quando da un qualsiasi oggetto riusciamo ad astrarre una sua qualità in modo da utilizzarla per un secondo fine ( esempio lampante è l'utilizzo di ocra nelle sepolture del Paleolitico superiore) esso diventa simbolo. A questo punto possiamo dire che si definisce un'opera d'arte La produzione di una qualunque espressione formale simbolica non utilitaristica, per mezzo della quale sia stata lasciata una traccia durevole sulla materia (De Marinis 2006). Da queste supposizioni si può comprendere uno degli obiettivi principali della ricerca, ossia, contribuire allo studio delle Veneri Paleolitiche in maniera scientifica, e quindi oggettiva, ampliando il limite interpretativo e talvolta soggettivo che le analisi estetico-tipologiche forniscono in un campo legato a culture lontane dal nostro presente. Impresse, indelebili e inequivocabili sono, infatti, le tracce tecnologiche, lasciate sulle Veneri dagli artisti paleolitici e, la loro analisi, condotta attraverso un protocollo sperimentale permette di raggiungere tale scopo. Il protocollo sperimentale utilizzato in questa ricerca che, ha come fine, la comprensione di come e con quali azioni le Veneri paleolitiche furono realizzate, è composto da quattro macro-fasi divise in ulteriori micro-fasi:

1. Primo approccio al Campione Archeologico (Venere Archeologica presa in esame):

- Conoscenza del Campione Archeologico da analizzare (materiale, forma e dimensione).

- Informazioni di tipo tafonomiche.

- Informazioni sul campionario litico rivenuto nel sito archeologico preso in esame.

- Riconoscimento delle tracce tecnologiche maggiormente evidenti sul Campione Archeologico.

2. Creazione di una collezione di confronto: oggetti Campione (in osso):

- Creazione di un database Excel con voci riguardanti le informazioni da trarne sui Campioni.

- Acquisizione di 3 ossa lunghe animali conservate nel laboratorio LTFAPA della Sapienza corrispondenti a: Suino, Bovino, Ovino.

- Realizzazione del campionario litico affine al sito Archeologico preso in considerazione.

- Creazione oggetti Campione utilizzando la tecnica dell'abrasione, raschiatura e incisione alternando ossa e strumenti differenti.

- Analisi microscopiche delle tracce tecnologiche realizzate durante la creazione degli oggetti Campione. 
- Realizzazione del vocabolario tipologico mediante osservazione al microscopio delle tracce tecnologiche.

- Acquisizione dei dati ottenuti nel database Excel.

3. Creazione della Venere Campione:

- Acquisizione di un osso lungo di Bovino (inerente al Campione Archeologico in esame).

- Utilizzo della percussione diretta per l'ottenimento di un frammento d'osso di dimensioni simili al Campione Archeologico.

- Realizzazione del campionario litico affine al sito Archeologico preso in considerazione.

- Realizzazione della tecnica di abrasione, raschiatura e incisione per realizzare le varie parti anatomiche delle Venere Sperimentale tenendo in considerazione il Campione Originale.

- Analisi microscopiche delle tracce tecnologiche ottenute.

- Acquisizione dei dati ottenuti nel database Excel utilizzando il vocabolario tipologico delle tracce tecnologiche creato in fase sperimentale.

4. Confronto tracce tecnologiche: Venere Archeologica e Venere Campione:

- Analisi al microscopio delle tracce tecnologiche della Venere Archeologica.

- Confronto tracce tecnologiche Sperimentale e Originali al microscopio.

- Messa a punto del "Matrix Gestuale".

La Venere Archeologica di riferimento in questa ricerca è la "Venere Grande", chiamata anche la "Femme au voile" ossia la donna con il velo (Zampetti 2004), trovata nella Grotta di Parabita in Puglia datata presumibilmente tra il Gravettiano finale e il Epigravettiano iniziale (Radmilli 1969) ossia tra i 22000 e i 20000 BP. La perplessità concernente la sua datazione è dovuta alla sua dubbiosa posizione stratigrafica in quanto ritrovata in giacitura secondaria all'esterno della Grotta di Parabita (Piscopo, 1965). Una delle ipotesi proposte è che, la statuina fosse stata deposta all'interno della Grotta e accanto ad una sepoltura bisoma. Le concrezioni sulla superficie della Venere presentavano, infatti, il medesimo terreno dello strato di sepoltura bisoma (Cremonesi, 1992). Il materiale su cui è stata realizzata corrisponde ad un osso lungo attribuibile alla specie Bos primigenius (Zampetti et Alhaique 2004), che rappresenta una percentuale significativa della fauna presente nel sito. In base all'analisi archeozoologica si è stabilito che si tratta di un frammento della zona sinistra del femore in quanto il tessuto spugnoso dell'osso compare nel lato destro della statuina.
La Venere è di tipo a tutto tondo, con il capo allungato, l'assenza di elementi facciali (non sono presenti, naso, bocca, occhi) sostituiti da tre fasce parallele che sembrano formare un velo o un turbante, seno pendente e fianchi espansi, le gambe terminano a punta e sono assenti i piedi. Attualmente la Venere Grande è conservata nel Museo M.A.R.T.A di Taranto (Puglia).

La prima fase della ricerca è stata la creazione di una serie di tracce tecnologiche effettuate su campioni d'osso, la "collezione di confronto", in modo da ottenere un sufficiente numero di elementi da confrontare con le tracce individuabili sulla Venere Archeologica. Le tracce tecnologiche sono quelle impronte che lasciano gli strumenti litici, ossei, metallici sulla materia lavorata. In base ad una loro analisi è possibile risalire alla produzione e all uso dell'oggetto indagato. Ogni traccia Campione ottenuta, è stata fotografata allo stereomicroscopio (Nikon, con obiettivi $10 \mathrm{X}$, oculare $0,5 \mathrm{X}$, a tre diversi ingrandimenti 0,75X/2X / 4X. Presso il laboratorio LTFAPA dell'Università di Roma "La Sapienza") e catalogata in base alla morfologia delle strie ottenute con la sperimentazione all'interno di un database con una specifica sigla. La seconda fase è stata la creazione di una Venere Campione, ossia la realizzazione di una statuina sperimentale in osso con dettagli anatomici simili alla Venere Archeologica utilizzando repliche di strumenti litici di alcuni materiali rinvenuti nel sito di Parabita. La Venere Campione è utile per ampliare la collezione di confronto e per poter osservare ancor più da vicina la gestualità del mondo artistico paleolitico. Le tracce tecnologiche della Venere Campione e della collezione di confronto si possono riassumere con l'esecuzione di tre gesti sperimentali: l'incisione attraverso l'uso di un bulino, la raschiatura effettuata con un raschiatoio con e senza manico e l'abrasione eseguita con pietra arenaria compatta e a tessitura fine. Le tracce tecnologiche della "Venere Campione" e della "Collezione di Confronto" sono state poi confrontate con la Venere Archeologica. Il confronto ha portato ad una ipotetica ricostruzione circa i gesti, i tempi e gli strumenti utilizzati dagli uomini paleolitici per la realizzazione della Venere, un vero e proprio "Matrix gestuale". La fase sperimentale che prevede, come da protocollo, la realizzazione di tracce tecnologiche è la loro identificazione è stata il secondo fine della ricerca. Si è cercato di creare ex novo una terminologia tipologica con identificazione traccia-azione senza sfruttare il vocabolario di tipo tradizionale utilizzato dai maggiori studiosi in materia. L'obiettivo è quindi capire se un vocabolario immediato, 
in quanto ottenuto dalla semplice osservazione descrittiva delle tracce, potesse essere applicato al campo dell'arte e se fosse funzionale alla ricostruzione del "Matrix gestuale".

\section{IL DATABASE: LA CREAZIONE DELLA “COLLE- ZIONE DI CONFRONTO"}

Il database realizzato è stato effettuato con il programma Excel inserendo delle voci che forniscono informazioni sui campioni utilizzati. Il database è stato diviso in tre grandi campi a loro volta suddivisi in sotto-campi: 1) Informazioni sul Campione.

2) Informazione sugli strumenti da lavoro.

3) Lavoro effettuato e risultati finali.

I campioni sono stati denominati con un codice alfanumerico del tipo $1 \mathrm{~A} 1$ dove la prima cifra indica il numero di Campione, la lettere, la superficie dell'osso utilizzata nella sperimentazione e la seconda cifra, può essere accompagnata da una lettera minuscola che ne indica il numero di esperimento (su una stessa superficie possono essere effettuate due sperimentazioni). Seguono informazioni circa la specie animale utilizzata, la parte anatomica e sua morfologia, lo stato di conservazione del Campione (in cenere, congelato) e la dimensione dell'osso. Gli strumenti da lavoro utilizzati sono stati inseriti specificando: la materia prima utilizzata (pietra/selce), tecnologia (blocco/lama), il tipo di strumento (abrasiva dura e sabbiosa, raschiatoio, bulino), se è presente o meno il ritocco, le dimensioni dello strumento in centimetri e l'assen$\mathrm{za}$ /presenza del manico ( $\mathrm{e}$ in tal caso la sua larghezza o lunghezza totale). L'ultimo campo stabilisce i modi e i gesti del lavoro effettuato, indicando, in primo luogo, il tipo di azione eseguito (abrasione, incisione e raschiatura) e il verso nel quale si è agiti. L'azione può essere del tipo unidirezionale, un gesto condotto in un solo verso che può essere sinistra-destra (S-D) o viceversa, o dall'alto verso il basso (A-B) o viceversa, e del tipo bidirezionale, un movimento senza interruzione S-D; D-S oppure A-B; B-A. Per chiarezza è importante precisare che quando ci si riferisce ad un'azione di tipo unidirezionale intendiamo un movimento che parte da un punto A e si ferma ad un punto B, e quindi, per poter ricompiere la medesima azione dobbiamo ritornare sul punto A. Nel gesto bidirezionale invece, l'azione si svolge sempre partendo da un punto $\mathrm{A}$ e giungendo ad un punto $\mathrm{B}$, ma, per ricalcare il medesimo movimento si riparte dal punto $\mathrm{B}$ (un'azione quindi continua). Questo tipo di azione è ovviamente effettuata in un punto specifico del Campione che viene chiarito nella voce "localizzazione", l'area o la parte sul quale si è agito. Per poter identificare le tracce prodotte dai gesti compiuti sui campioni, è stato utile creare un vocabolario tipologico in modo che la traccia, fosse facilmente identificabile. Per cui le strie sono identificate da tre tipi di informazioni: le dimensioni, se sono Lunghe o Corte, in cui le prime, essendo più sottili, sono generalmente visibili ad ingrandimenti maggiori; lo spessore, ovvero se sono strie Profonde o Poco Profonde; e la loro morfologia che può essere $\mathrm{a} \mathrm{V}$, a U, oppure OV (quest'ultimo tipo è riproducibile solo durante l'azione di raschiatura). Accanto ad una prima identificazione delle strie spesso possono essere riconosciuti due simboli: il ">" significa che le strie indicate alla sinistra della freccia sono coperte dalle strie alla destra della stessa; "=" indica che le strie in entrambe i lati sono sullo stesso piano e quindi nessuna copre l'altra. La tipologia viene poi ulteriormente ampliata dalla voce "distribuzione", vale a dire la disposizione delle strie createsi durante la sperimentazione, se Coprono o Non Coprono del tutto la superficie e se hanno un andamento Ordinato o Disordinato. Nel testo sono state attribuite alle tracce tecnologiche le definizioni di "strie" o "solco" (nel caso della raschiatura) evidenziando un mancato utilizzo della terminologia tipologica classica che prevede, oltre ad un vocabolario standard azione-traccia (es. stria-abrasione, solco-incisione), anche, l'aggiunta dell'uso dei termini di politura, scanalatura e stigmate (Camps-Fabrer 1977; D'Errico et al. 1985; Averbouh 2000; Petillon 2006). Il mancato utilizzo di tal terminologia è dovuto ad un elaborazione di un vocabolario creato in laboratorio, e quindi in via del tutto sperimentale, di tipo semplificato e finalizzato a descrivere la forma delle tracce visibili al microscopio. La creazione di un vocabolario tipologico è stato, infatti il secondo fine di questa ricerca in quanto improntato a stabilire la sua possibile utilità in campo di tipo artistico. Per cui il termine "stria" viene attribuito a tutti i tipi di tracce oggettivamente visibile al microscopio, le cui caratteristiche dimensionali e morfologiche sono descritte dai termini sopra citati, mentre, il termine "solco" è stato utilizzato solo quando, applicata la tecnica di raschiatura si denotano strie di forma sferica o scanalate. Negli esperimenti si è impiegato un tempo standard di 30 minuti, tuttavia è preferibile utilizzare ugualmente la voce perché le schede riguardanti la produzione della Venere Campione sono le medesime e il tempo, in questo ultimo caso, varia. 


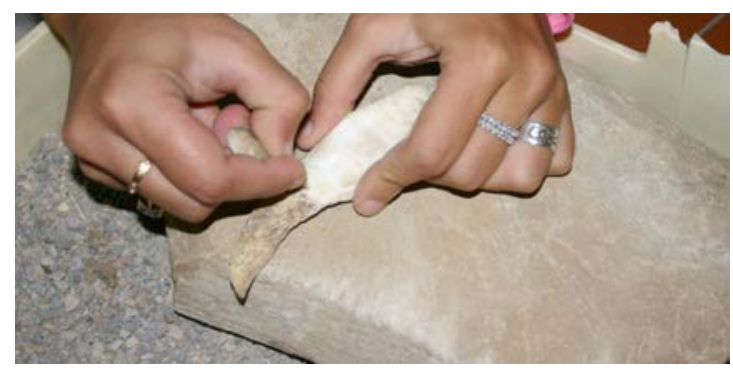

Fig. 1: Riproduzione gesto dell'incisione (M.Basile).

Prima di passare ad una breve rassegna del tipo di tracce tecnologiche prese in esame è bene sottolineare cosa si intende per abradere, incidere e raschiare. L'incisione (fig.1) è una traccia lasciata da un oggetto appuntito, in tal caso il bulino, sulla superficie dell'oggetto attraverso un gesto netto e deciso per lo più unidirezionale. L'abrasione (fig. 2) invece, consiste, nello sfregare l'oggetto che si desidera lavorare su o con una superficie ruvida, in tal caso un blocco di arenaria o un frammento di arenaria ed è generalmente un gesto bidirezionale. La raschiatura consiste, invece, nel lisciare la superficie presa in esame, attraverso un oggetto ruvido o tagliente, in tal caso un raschiatoio con margini dentellati, secondo un gesto per lo più unidirezionale.

Sono state utilizzate 3 ossa lunghe: di suino, ovino e bovino si sono applicate i gesti di raschiatura, abrasione e incisione lungo le loro superfici per un totale di 7 sperimentazioni differenti. La limitata scelta corrispondente alle sole ossa lunghe di tali specie animali è stata dettata, dalla disponibilità di Campioni offerti al momento della sperimentazione all'interno del laboratorio LTFAPA, Dipartimento di Scienze dell'Antichità, Università di Roma "Sapienza", dove è stata realizzata questa ricerca. Le ossa lunghe erano conservate sotto cenere o congelate, per cui, il loro utilizzo ha richiesto in un primo momento l'ausilio di acqua calda per pulire la superficie e di una seconda fase di breve asciugatura per iniziare la sperimentazione. Anche tutte le fasi sperimentali e le osservazioni al microscopio sono state effettuate nel medesimo laboratorio.

\section{CAMPIONE 1A1- 1A1a}

Il primo Campione, $1 \mathrm{~A}$, corrisponde all' area interna della diafisi di un osso di suino di dimensione $15,3 \times 3$ $\mathrm{cm}$ e conservato sotto cenere. Nel primo esperimento,
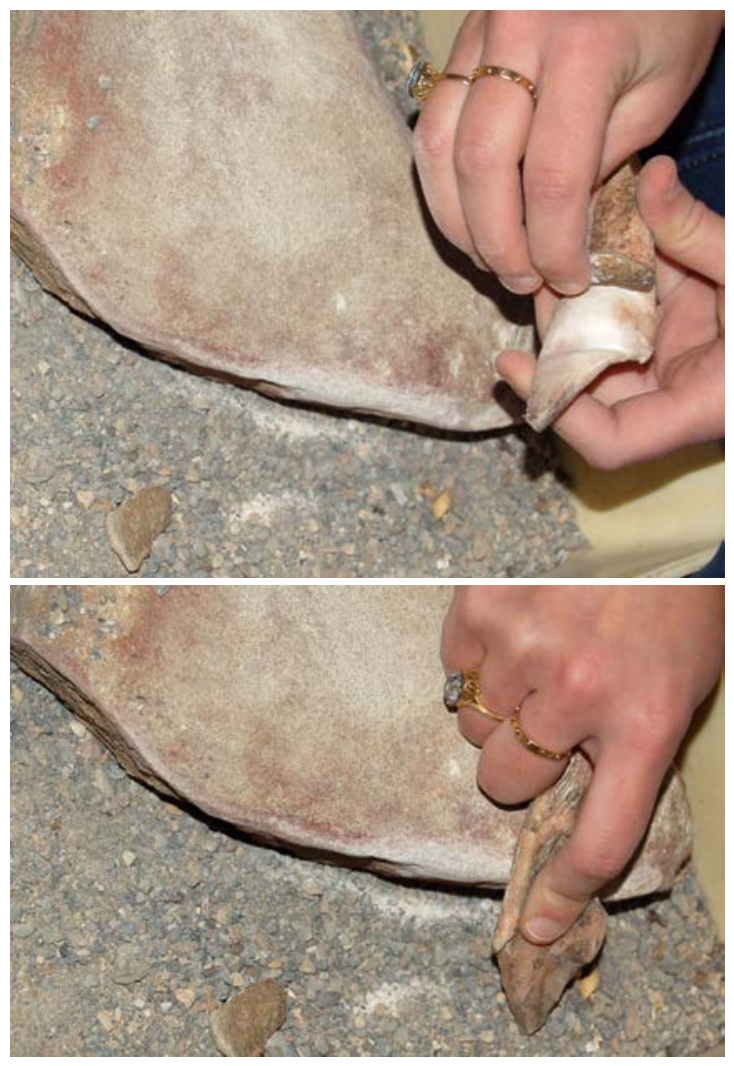

Fig. 2: Riproduzione gesto dell'abrasione. A: su blocco di arenaria duro; B: con frammento di arenaria duro (M. Basile).

1A1, (fig.3A) l'osso è stato prelevato dalla cenere, è stato lavato e fatto asciugare. Dopo la pulizia è stato prelevato e abraso su un blocco di arenaria sabbiosa con un movimento bidirezionale dall'alto verso il basso (A-B/B-A) per 30 minuti senza interruzione. Alla fine è stato analizzato al microscopio. Sulla superficie sono state osservati due tipi di strie, le prime risultano lunghe, poco profonde con morfologia a "V" (L-PP-V) con distribuzione coprente e disordinata (CO-D) e sono coperte $(>)$ da strie corte, profonde con morfologia ad "U" (C-P-U) con distribuzione non coprente e ordinata (NC-O).Sullo stesso Campione (1A) e con lo stesso blocco di arenaria si è provveduto all'abrasione bidirezionale del lato spugnoso collocato a sinistra dell'osso, esperimento 1A1a (fig. 3B). Dopo trenta minuti è stata terminata l'operazione. Lo scopo era quello di stabilire, se l'abrasione fatta con strumenti litici identici su due superfici diverse (parte spugnosa e area della diafisi interna dell'osso), producesse strie morfologicamente 

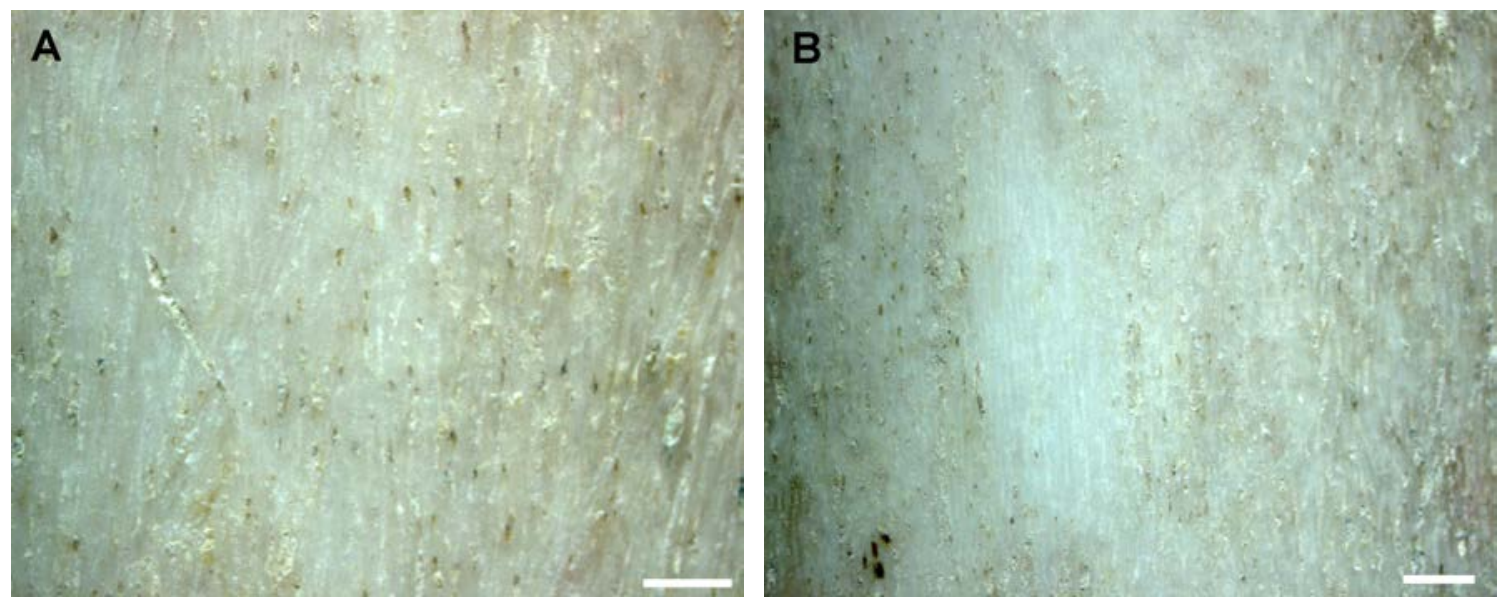

Fig. 3: A: Esperimento 1A1, abrasione bilaterale, con blocco di arenaria sabbioso ingrandimento 4x=4mm; B: Esperimento 1A1a, lato spugnoso osso, abrasione bilaterale, con blocco di arenaria sabbioso ingrandimento $2 \mathrm{x}=2 \mathrm{~mm}$ (M. Basile).

disuguali. La sperimentazione ha dato esito negativo, in quanto, le strie prodotte sul Campione $1 \mathrm{~A} 1$ e $1 \mathrm{~A} 1 \mathrm{a}$ erano analoghe.

\section{CAMPIONE 2A1-2A1a}

Il secondo Campione, 2A, è invece la parte interna della diafisi di un osso di ovino di dimensione $12 \times 2 \mathrm{~cm}$ e conservato sotto cenere. Dopo la pulizia è iniziata la sperimentazione, 2A1, (fig. 4) con l'abrasione del Campione sulla superficie di un blocco di arenaria compatta secondo un movimento bidirezionale A-B/B-A. Dopo 30 minuti si è provveduto all'analisi al microscopio. Le strie ottenute corrispondo alla tipologia lunghe, poco profonde, con morfologia ad "U" (L-PP-U)) con distribuzione coprente e disordinata (CO-D). La seconda sperimentazione, 2A1a, (fig. 4a) era invece mirata alla efficacia della abrasione effettuata con la pietra arenaria dura, per cui, prendendo in considerazione una naturale deformazione dell'osso che risultava leggermente rigonfio nella zona destra del Campione, si è effettuata una abrasione bidirezionale per 30 minuti lungo tale superficie. Alla fine della sperimentazione la deformazione naturale era totalmente scomparsa (fig. 4b).

\section{CAMPIONE 3A1- 3B1- 3B1a}

Il Campione 3 è stato lavorato su entrambe le superfici anatomiche (A-B) e corrisponde ad un femore di bovino provvisto di periostio di dimensione $12 \times 4$ $\mathrm{cm}$, conservato congelato. Prelevato il Campione lo si è successivamente messo in acqua calda per circa dieci minuti e fatto asciugare. Dopo questa operazione è iniziata la sperimentazione, 3A1, (fig. 5A). Con un raschiatoio in selce senza manico si è raschiata la superficie della parte carnosa, procedendo con un movimento unidirezionale dall'alto verso il basso (AB). Dopo 30 minuti si è passati all'analisi delle tracce tecnologiche . Le strie risultavano di due tipologie disposte sullo stesso piano (=), lunghe, poco profonde con morfologia a "V" (L-PP-V) con distribuzione non coprente e ordinata (NC-O) e strie corte, profonde con morfologia "OV" (C-P-OV) con distribuzione coprente e ordinata $(\mathrm{C}-\mathrm{O})$. La morfologia "OV" è stata chiamata "solco a catena" a causa dell'aspetto stesso della stria. Il secondo esperimento è stato effettuato sulla superficie opposta dello stesso Campione, 3B1, (fig. 5B) lo scopo era quello di verificare l'efficacia della raschiatura unidirezionale (A-B) con raschiatoio provvisto, questa volta, di manico. Le strie in tal caso risultano lunghe, profonde con profilo ad "U" con distribuzione coprente e ordinata. In relazione alla loro morfologia queste strie sono state chiamate "Solchi a scalino". L'ultima sperimentazione è stata effettuata sulla superficie raschiata con un raschiatoio immanicato, 3B1a, (fig. 5b) .L'incisione è stata effettuata con un bulino senza manico con movimento bidirezionale sinistra, destra (S-D/D-S). Le tracce corrispondono alla morfologia corte, poco profonde, con morfologia $\mathrm{a}$ "V" (C-PP-V) con distribuzione poco coprente e disordinata (PC-D). 


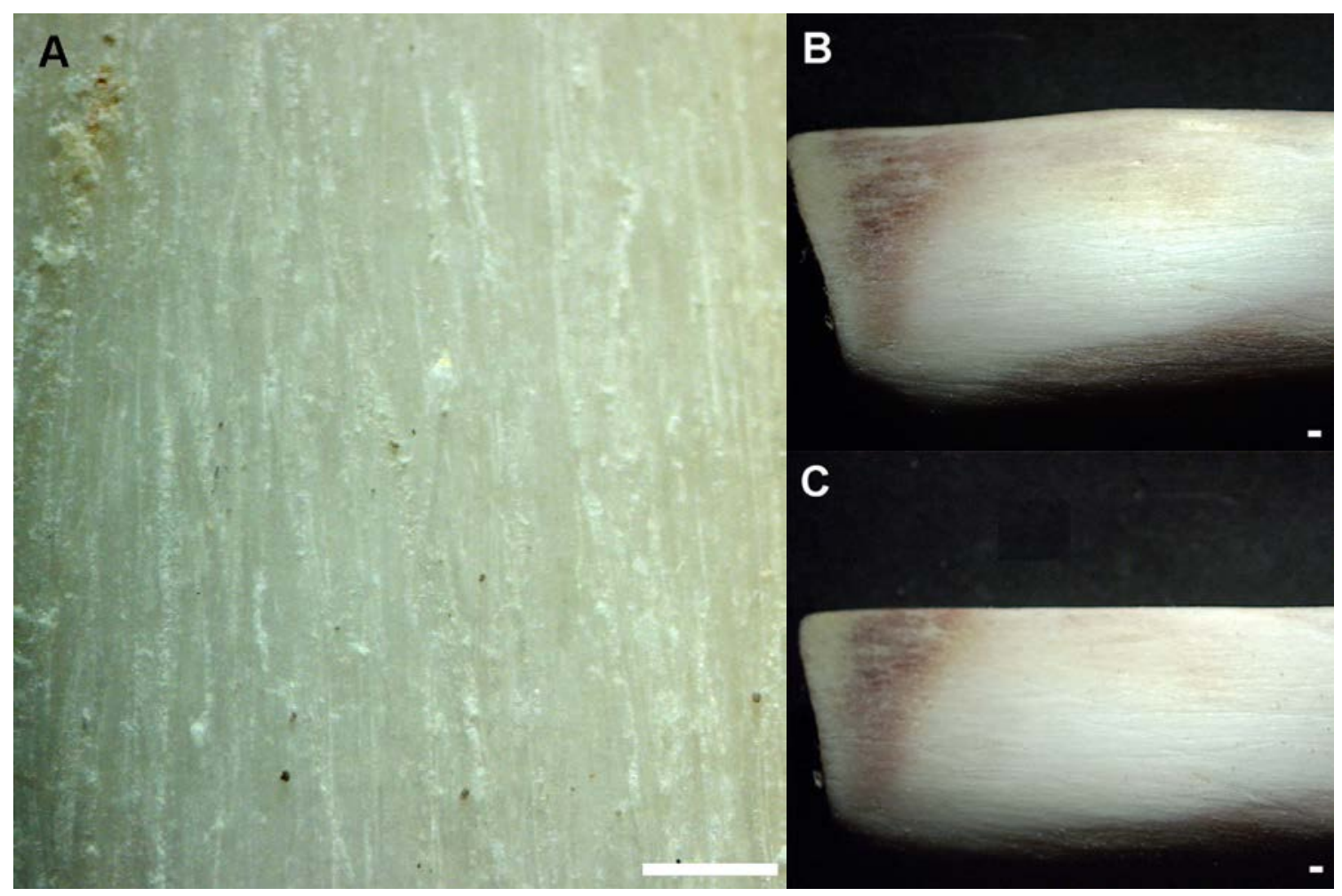

Fig. 4: A: Esperimento 2A1, lato concavo osso, abrasione bilaterale, con blocco di arenaria duro ingrandimento 4x=4mm; B: Esperimento 2A1a, lato deformato naturalmente, abrasione bilaterale, con blocco di arenaria dura ingrandimento 0,75x; C: Esperimento 2A1a, lato deformato naturalmente, dopo 30 minuti di abrasione bilaterale, con blocco di arenaria duro ingrandimento 0,75x (M. Basile).
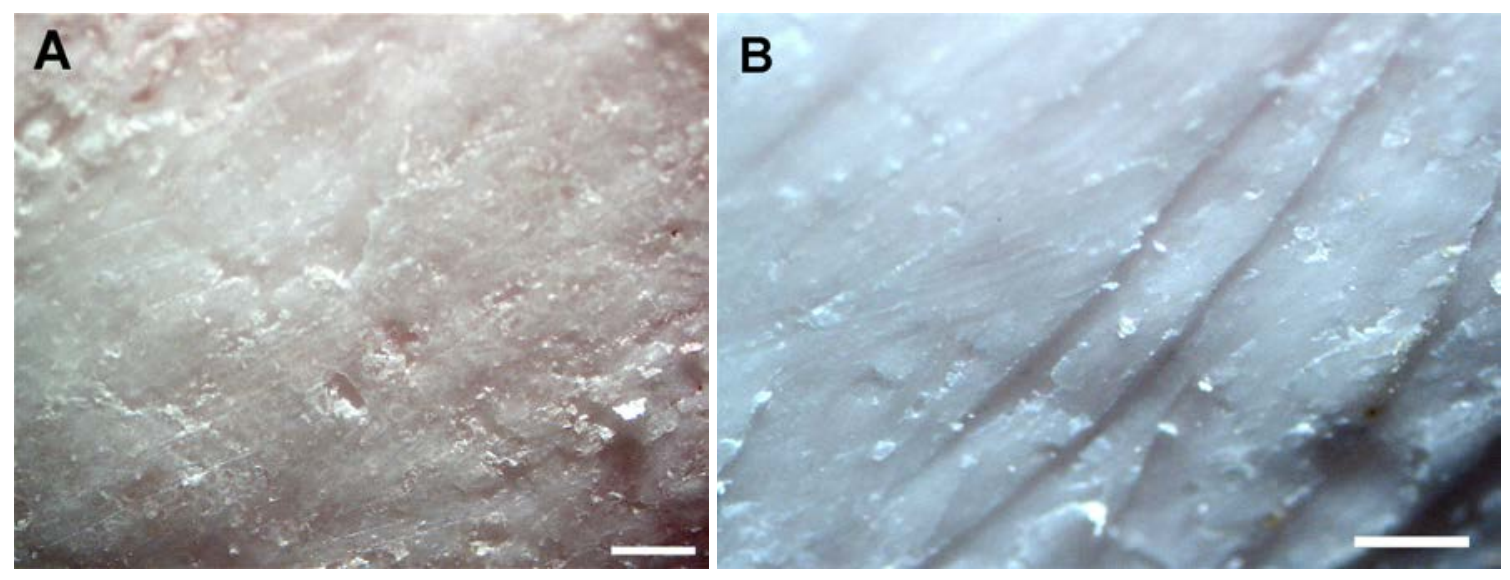

Fig. 5: A. Esperimento 3A1, raschiatura unilaterale, ingrandimento 4x=4mm; B: Esperimento 3B1, raschiatura unilaterale con raschiatoio immanicato, ingrandimento $4 \mathrm{x}=4 \mathrm{~mm}$ (M. Basile).

Le sperimentazioni hanno portato ad alcune considerazioni preliminari circa l'efficacia di alcuni strumenti a discapito di altri e sul tipo di gestualità da utilizzare:
- L'abrasione con pietra abrasiva dura risulta maggiormente adeguata per una lavorazione precisa e sbrigativa dell'osso. L'insorgenza, infatti, di una minor quantità 


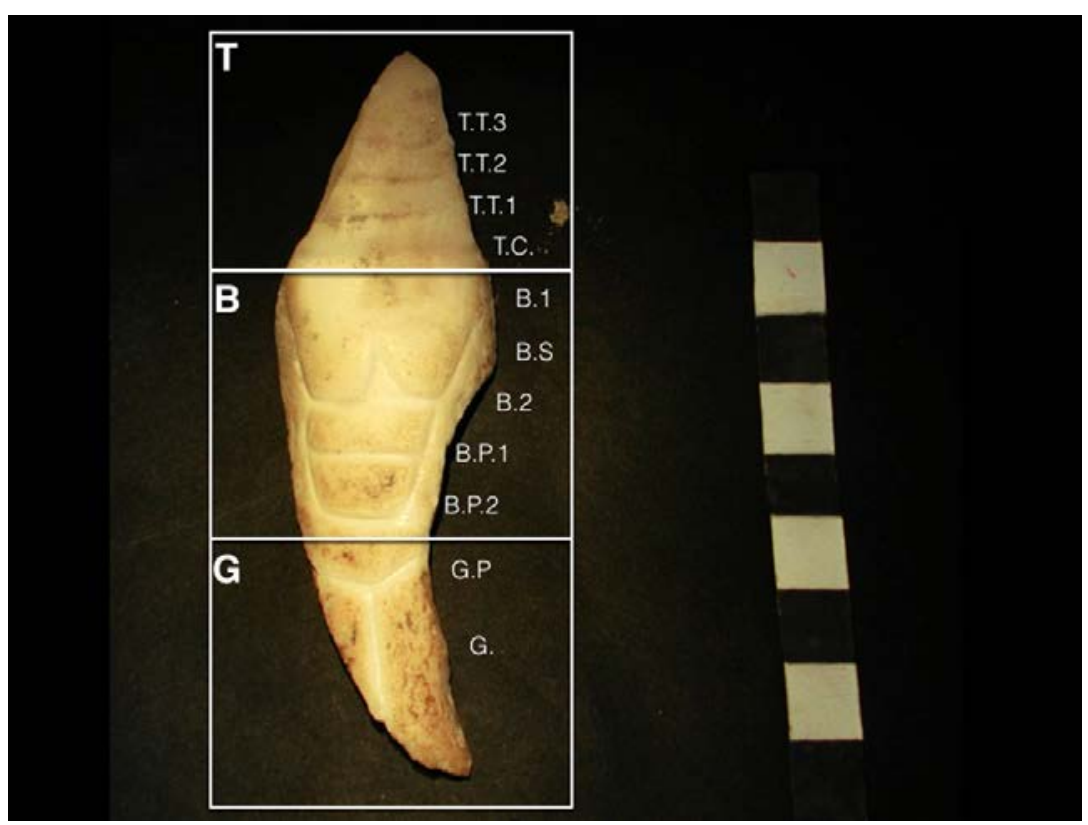

Fig. 6: Venere Campione, divisione anatomica sperimentale presa come riferimento (M. Basile).

di strie e il veloce cambiamento morfologico dell'osso è visibile nell'esperimento 2A1a.

- La raschiatura risulta molto utile per l'eliminazione del periostio dell'osso, in soli 30 minuti, si è reso l'osso liscio e pronto per essere inciso. Il manico applicato al raschiatoio rende ancora più veloce e precisa questa operazione. Le strie lasciate dalla raschiatura inoltre sono facilmente visibili e leggibili al microscopio.

- L'incisione effettuata con bulino risulta molto facilitata se attuata su superfici già abrase o raschiate. Il miglior procedimento per effettuare incisioni precise risulta quello di creare un unica stria sottile e poi con un movimento bidirezionale S-D di allargarne l'ampiezza.

Dopo la creazione della collezione di confronto si è provveduto alla realizzazione della "Venere Campione".

\section{LA "VENERE CAMPIONE"}

Il supporto utilizzato per realizzare la Venere è stato ottenuto dal materiale di scarto (debitage) a seguito della frantumazione di un osso di bovino tramite percussione diretta con un nucleo di selce. La scelta è ricaduta su un frammento che si avvicinava, in termini dimensionali e morfologici, alla Venere Grande archeologica presa come riferimento per la sperimentazione. Il frammento osseo preso in considerazione misurava $11 \mathrm{~cm}$ di lunghezza e $4 \mathrm{~cm}$ di larghezza, differendo dall'originale già lavorata di 3 $\mathrm{cm}$ per la lunghezza e di $2 \mathrm{~cm}$ per la larghezza. La Venere originale risulta, infatti, dopo la sua lavorazione, di $9 \mathrm{~cm}$ di lunghezza e $2 \mathrm{~cm}$ di larghezza. Non possiamo escludere che precedentemente la lavorazione l'osso era di maggiori dimensioni. Al fine di rispettare quanto più possibile l'estetica della Venere Archeologica nella suddivisione delle linee di incisione, è stata applicata la seguente proporzione che tenesse conto delle differenze dimensionali:

9( $\mathrm{cm}$ Venere originale $): 11(\mathrm{~cm}$ Venere campione $)=2,27$ (cm di lunghezza del Testa Venere originale): $x$

Per poter facilitare il lavoro di riproduzione della Venere Campione mi è sembrato utile dividere la Venere Archeologica in tre parti: Testa (T.), Busto (B) e Gambe (G). Prese le misure si è effettuata la stessa operazione direttamente sul Campione creando una prima suddivisione di queste tre aree (tenendo in considerazione la proporzione). Il risultato ottenuto è stato che la testa della Venere Campione dovesse interrompersi intorno ai $2,8 \mathrm{~cm}$ di lunghezza, il Busto intorno ai $7,4 \mathrm{~cm}$ li lunghezza e il centimetro restante fosse utilizzato per le Gambe. Dopo questa prima operazione, le tre zone principali sono state ulteriormente divise in base alle linee di incisione interne alla Venere Archeologiche. Le linee di incisione interne sono state definite con l'iniziale della zona di appartenenza 
( $\mathrm{T}=$ testa, $\mathrm{B}=$ busto, $\mathrm{G}=$ gambe) accompagnate da un numero o da una lettera. Ogni esperimento è stato poi inserito all'interno di un database simile a quello precedentemente presentato. Il lavoro è stato eseguito, senza prima raschiare la superficie, partendo dalla realizzazione del collo, per poi passare alla zona del Busto, della Testa e in ultimo delle Gambe.

Si precisa che non è stato eseguita la zona dei glutei in quanto lo spessore della Venere Campione, risultava poco adatto per un lavoro a tutto tondo. La parte lavorata è quindi solo su un lato dell'osso. All'interno del database la Venere Sperimentale corrisponde al Campione 4 e il numero di esperimento sta ad indicare i giorni impiegati per la lavorazione dell'osso (4A1g, 4A2g ecc.). Gli strumenti utilizzati per la realizzazione della statuina sono attribuibili ad un unico bulino ritoccato e ad un frammento e un blocco di pietra arenaria dura. Il blocco di arenaria spesso è stato utilizzato anche come base d'appoggio per incidere con il bulino e abradere con il frammento di arenaria, in tal caso è segnato con il simbolo asterisco $(*)$. Le informazioni sulla tipologia e la distribuzione delle tracce sono state inserite e discusse alla fine del lavoro sperimentale in base alle foto eseguite al microscopio. Questo perché la realizzazione della Venere è avvenuta in più giorni, nel quale spesso accanto all'obiettivo del giorno, precedeva un lavoro di ravvivamento delle incisioni effettuate precedentemente. Ne risulta che la tipologia delle strie, su ogni "parte lavorata", è stata fatta alla fine del lavoro sperimentale e tenendo conto di tutte le operazioni effettuate attraverso un "matrix gestuale", ossia un riassunto schematizzato delle azioni effettuate su ogni incisione. Si ricorda che, il tempo di realizzazione di ciascuna linea incisa o parte lavorata prende in considerazione anche lavorazioni avvenute in giorni differenti dalla prima realizzazione. Il lavoro effettuato è stato qui schematizzato tenendo in considerazione le tre aree principali della Venere, partendo dalla Testa, per poi passare al Busto e alle Gambe. Le foto sono state eseguite mediante uno stereomicroscopio Nikon, con obiettivi 10X, oculare $0,5 \mathrm{x}$, a tre ingrandimenti diversi $\mathrm{x} 1, \mathrm{x} 2$ e x 3 .

\section{CAMPIONE: 4A1g-4g, LA TESTA}

La zona della testa presenta un totale di 4 tracce tecnologiche identificate dal codice alfa numerico T.T.3, T.T.2, T.T.1 e T.C. Le lettere iniziali stanno per "tagliotesta", il numero per le incisioni e nell'ultimo caso la C. indica il collo.
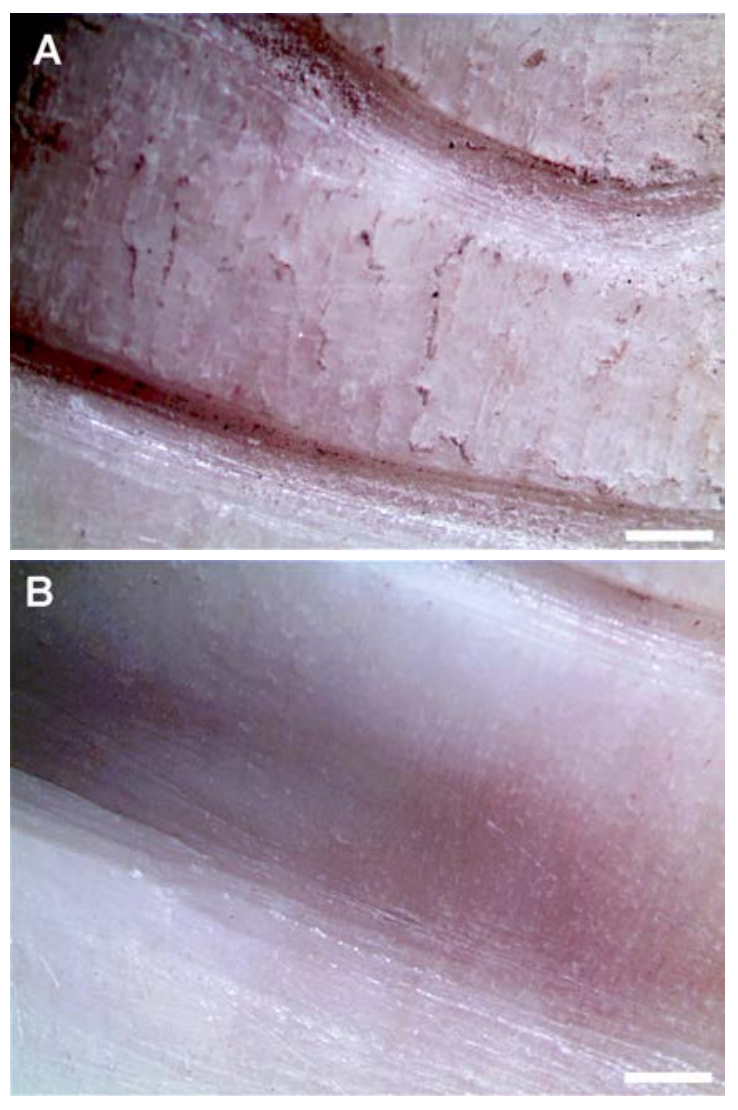

Fig. 7: A: Venere Campione, strie parte anatomica T.T.3 e T.T.2, incisione con bulino, ingrandimento $2 \mathrm{x}=2 \mathrm{~mm}$; B: Venere Campione, strie parte anatomica T.C., abrasione con frammento e blocco di arenaria duro alternato all'uso di bulino per l'incisione, ingrandimento $2 \mathrm{x}=2 \mathrm{~mm}$ (M. Basile).

Le prima incisione effettuata è stato il taglio T.T.3 (4A1g; fig.7A) realizzata attraverso l'azione bidirezionale S-D/D-S e appoggiando il Campione su un blocco di arenaria $(*)$. Il tempo impiegato è stato di circa 35 minuti di lavoro e si sono prodotte strie del tipo lunghe, poco profonde con morfologia ad "U" (L-PP-U) con distribuzione poco coprente ed ordinata (PC-O). Il taglio T.T.2 $(4 \mathrm{~A} 2 \mathrm{~g})$ è stato eseguito allo stesso modo, ossia, l'uso del bulino con gesto bidirezionale e appoggiando il Campione su blocco d'arenaria, se ne deduce che si sono prodotte le medesime strie con un tempo di 37 minuti. Il taglio T.T.1 $(4 \mathrm{~A} 3 \mathrm{~g})$, invece, è stato effettuato con l'assenza del blocco di arenaria come base di appoggio per un tempo di 37 minuti. Le strie risultano con la medesima morfologia ad "U" ma maggiormente profonde e coprenti. Il Taglio T.C.(4A4g; fig. 7B) che corrisponde alla zona del collo 


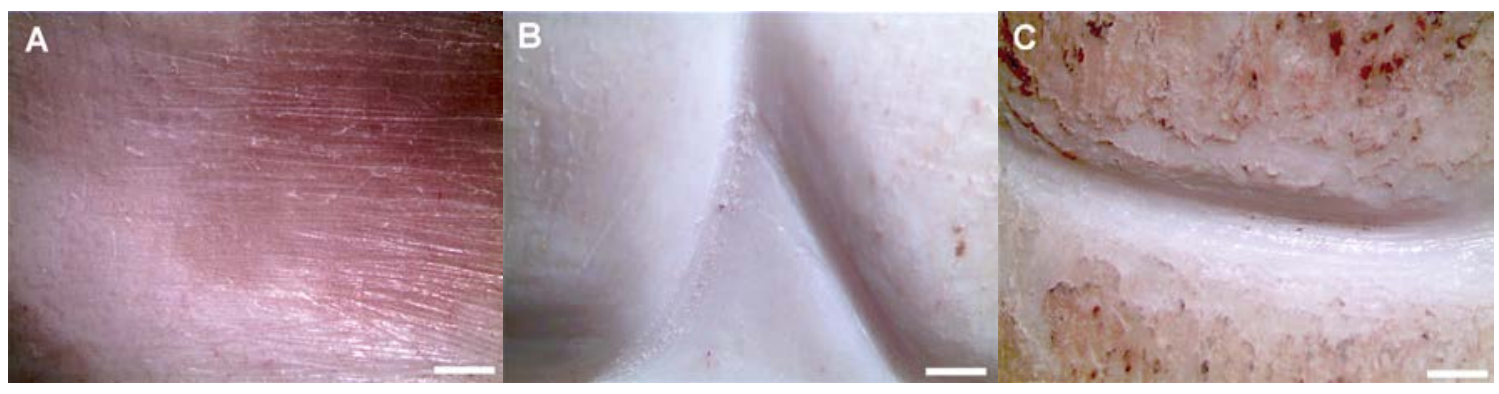

Fig. 8: A: Venere Campione, strie parte anatomica B.1, abrasione con frammento e blocco di arenaria duro, ingrandimento $2 \mathrm{x}=2 \mathrm{~mm}$; B: Venere Campione, strie parte anatomica B.S e B.2, incisione con bulino e abrasione con frammento di arenaria duro, ingrandimento $2 \mathrm{x}=2 \mathrm{~mm}$; C: Venere Campione, strie parte anatomica B.P.2, incisione con bulino e abrasione con frammento di arenaria duro, ingrandimento $2 \mathrm{x}=2 \mathrm{~mm}$ (M. Basile).

(Taglio Collo) è stato invece effettuato mediante una doppia azione combinata di tipo bidirezionale S-D/D-S. Si è iniziata, infatti, ad abradere la parte per un brevissimo periodo direttamente con il blocco di arenaria, facendo aderire la superficie della Venere Campione ad uno degli spigoli del blocco stesso. Successivamente si è incisa a lungo la superficie $(*)$ alternandola con l'aiuto di un frammento di arenaria dura . L'alternanza dei due strumenti ha facilitato il lavoro di resa tridimensionale della superficie. Dopo 90 minuti di lavoro si è proceduti all'analisi al microscopio. Le strie risultano lunghe, profonde con profilo a "V" distribuite in maniera poco coprente e disordinata.

La testa della Venere Campione è stata realizzata in un totale di 199 minuti di lavoro mediante l'utilizzo del bulino e della pietra arenaria dura.

\section{CAMPIONE: 4A5g-10g, IL BUSTO}

La zona Busto presenta un totale di 5 tracce tecnologiche, B.1 corrisponde all'area tra la linea del collo e la parte al di sopra del seno, B.S alle linee incise del seno, B.2 si riferisce alla parte abrasa sotto e fra il seno e B.P.1 e B.P.2 (busto, pancia) sono invece le incisioni addominali.

La zona B.1 (4A5g; fig. 8A) è stata realizzata attraverso l'unica tecnica dell' abrasione. Si è alternato un movimento bidirezionale sinistra-destra (S-D/D-S) con il frammento di arenaria duro e un abrasione sul blocco di arenaria di tipo bidirezionale dall'alto verso il basso (A$\mathrm{B} / \mathrm{B}-\mathrm{A})$. L'azione abrasiva è stata ripresa in più momenti anche durante la realizzazione delle zone B.S e B.2 per un totale di 120 minuti di lavoro. Le strie che si sono prodotte sono di due tipi lunghe, profonde con morfologie a "V" (L-P-V) e distribuzione coprente e disordinata (CO- D) sovrapposte (>) da strie corte, poco profonde con profilo a "V" e distribuzione poco coprente e disordinata (PC-D). Quasi in contemporanea si è realizzate l'incisioni del seno B.S (4A6g; fig. 8B) attraverso l'uso di un bulino ritoccato con movimento unidirezionale e appoggiando la statuina sul blocco di arenaria (*). Il tempo di realizzazione totale dell'area è stato di 135 minuti producendo strie del tipo lunghe, poco profonde e morfologia a "V" (L-PP-V) e distribuzione poco coprente e disordinata coperte $(>)$ da strie corte, poco profonde e profilo a "V" (C-PP-V) e distribuzione poco coprente disordinata (PC-D). Le tracce che coprono sono state prodotte dall'azione di abrasione inerenti all' area B.2. La zona abrasa B.2 (4A7g; fig. $8 \mathrm{~B})$ è stata realizzata attraverso l'alternanza di incisione con bulino di tipo bidirezionale (S-D/D-S) e abrasione con frammento di arenaria bidirezionale (AB/B-A). Le strie che si sono prodotte sono del tipo lunghe, profonde, con profilo a "V" (L-P-V) con distribuzione poco coprente e disordinata (PC-D). Il tempo impiegato è di 40 minuti. Le aree lavorate fino a questo punto sono state nuovamente ritrattate per altri 90 minuti con i medesimi strumenti in modo da risultare il più definite possibile. Questo ha consentito di aumentare la tridimensionalità della statuina creando zone più rotondeggianti. L'area B.1 non è stata ritrattata con bulino ma solo con un alternanza di blocco e frammento di arenaria dura. Le aree B.P.1 (4A9g) e B.P.2 (4A10g; fig. 8C) sono state trattate con bulino e frammento di arenaria duro, alternando quindi incisione e abrasione bidirezionale (S-D/D-S) e appoggiando la statuina sul blocco di arenaria duro (*). Il tempo di realizzazione totale delle due zone è stato di 85 minuti di lavoro (42 minuti B.P.1; 43 minuti B.P.2) e le strie prodotte 
corrispondono al tipo lunghe, poco profonde e con morfologia a "V" (L-PP-V) e distribuzione coprente ordinata $(\mathrm{CO}-\mathrm{O})$ coperte da tracce $(>)$ corte, profonde e profilo a "V" con distribuzione coprente e disordinata (CO-D).

Il busto è stato realizzato quindi mediante l'alternanza di abrasione e incisione per un tempo totale di 470 minuti.

\section{CAMPIONE: 4A11g, LE GAMBE}

L'area delle gambe presenta un totale di due tracce tecnologiche, G.P (fig. 9) ossia la zona pubica e G. le gambe con assenza di piedi. La realizzazione delle due aree è stata fatta allo stesso momento mediante un unica linea di incisione realizzata con l'azione del bulino in senso bidirezionale (S-D/D-S) e poi perfezionato con il frammento di arenaria duro. Entrambe le azioni sono state effettuate poggiando la statuina sul blocco di arenaria dura (*). Il tempo impiegato per la realizzazione di quest'area è di 57 minuti di lavoro e le strie prodotte sono del tipo lunghe, poco profonde, con morfologia a "V" (L-PP-V) con distribuzione coprente e ordinata (CO-O) sovrapposte da strie corte, profonde, con profilo a "V" (C-P-V) e distribuzione (CO-D).

L'intera statuina è stata realizzata in 638 minuti, la sua esecuzione, seppur abbozzata, è stata di media difficoltà. Osservazioni interessanti possono essere condotte circa gli strumenti utilizzati per la sua realizzazione. Il bulino, il frammento di arenaria e il blocco di arenaria, non sono mai stati sostituiti o ritoccati ma anzi sono stati funzionali per tutta la sperimentazione. Il blocco di arenaria è stato utile sia come strumento di abrasione soprattutto per le aree più ampie (B.1 e T.C), sia come supporto alla lavorazione rendendo le incisioni o le abrasioni con il frammento di arenaria dura più marcate. La tecnica dell'incisione è utile per la realizzazione dei tratti marcati e delle linee nette ma la tridimensionalità e il suo aspetto "a tutto tondo" la si raggiunge attraverso l'azione abrasiva. Per quanto riguarda le tracce tecnologiche si può dire che:

- Le strie provocate dalla sola incisione risultano lunghe, profonde e ad $\mathrm{U}$ con una disposizione coprente e ordinata. Nel caso in cui l'incisione è avvenuta con il blocco di arenaria usato come base, le strie diventano poco profonde e poco coprenti. La diminuzione delle strie potrebbe essere stata provocata dal fatto che il bulino non tocca unicamente di punta, quindi incidendo e ripassando più volte si ha l'eliminazione delle linee nette della incisione.

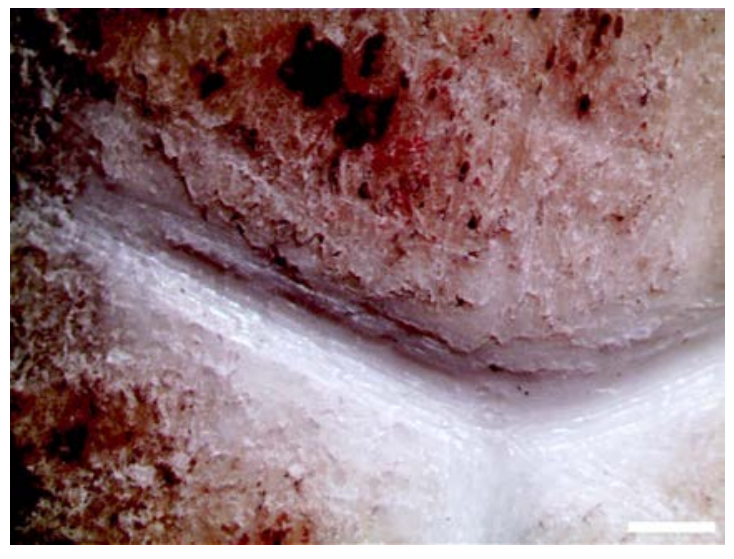

Fig. 9: Venere Campione, strie parte anatomica G.P, incisione con bulino e abrasione con frammento di arenaria duro, ingrandimento $2 \mathrm{x}=2 \mathrm{~mm}$ (M. Basile).

- Le strie provocate dalla sola abrasione risultano invece lunghe, profonde, a V e coperte da strie corte, poco profonde e a V. La doppia presenza di strie è dovuta all'azione di piccolissimi frammenti di arenaria che durante l'operazione di abrasione si staccano dagli strumenti.

- Le strie invece provocate dall'alternanza di abrasione e incisione risultano lunghe e a "V". Vi sono delle differenze riguardanti la profondità; le strie poco profonde, si producono quando si utilizza il bulino o il frammento di arenaria poggiando la Venere sul blocco, mentre il loro uso con assenza di appoggio produce strie profonde.

- La distribuzione delle strie sulla Venere Campione risulta ordinata sia se si utilizza il solo bulino con o senza appoggio sul blocco di arenaria, sia se si ha un'alternanza tra bulino e frammento di arenaria. Risultano invece strie disordinate unicamente quando compare nell'azione abrasiva anche il blocco di arenaria dura.

- In generale: l'incisione provoca delle strie di morfologia ad "U", l'abrasione provoca delle strie di morfologia a "V", mentre la loro azione combinata crea strie con morfologia a "V" associate ad ulteriori strie più piccole che coprono le prime.

La postura utilizzata durante la produzione della Venere è stata per lo più seduta a gambe incrociate e la lavorazione dell'osso con strumenti non tecnologici non risulta particolarmente difficile. Queste osservazioni suggeriscono che si trattava di un lavoro accessibili a tutti, uomini e donne, e a qualsiasi fascia di età, giovani, adulti, anziani. 


\section{IL CONFRONTO TRA L'ARCHEOLOGICO E LA SPERIMENTAZIONE}

Grazie all'autorizzazione ottenuta dal Museo M.A.R.T.A di Taranto si è proceduto all' analisi al microscopio delle tracce tecnologiche presenti sulla Venere Grande della Grotta di Parabita. L'analisi è stata eseguita attraverso una macchina fotografica Nikon montata direttamente sullo stereomicroscopio e con un microscopio USB digitale Dino-Lite. L'identificazione delle tracce tecnologiche è risultata alquanto difficoltosa a causa della presenza di Paraloid utilizzato per consolidare la superficie e a causa delle alterazioni post-deposizionali osservabili lungo le aree del capo, delle gambe e nella zona della schiena della Venere. Utilizzando la tipologia delle tracce tecnologiche ottenute dalla "collezione di confronto" e della "Venere Sperimentale" si sono potute effettuare le seguenti osservazioni, che per semplicità saranno riportate partendo dalla zona della testa, del busto e infine delle gambe. Si puntualizza inoltre che il nome utilizzato per identificare le incisioni e le abrasioni della Venere Sperimentali coincidono con le tracce tecnologiche identificate sulla Venere Archeologica.

Per quanto riguarda la zona della Testa: Le strie maggiormente visibili della Venere Archeologica sono quelle del taglio T.T.3 che mostrano tracce del tipo lunghe, profonde, con morfologia a "U" (L-P-U) e distribuzione non coprente e ordinata (N.C-O). Queste tracce sono confrontabili con le strie prodotte dall'uso del bulino con assenza del blocco di arenaria come base di appoggio (taglio T.T.1 Venere Sperimentale). L'assenza o la presenza di una base di appoggio è tuttavia valutabile solo attraverso la profondità delle strie. Se si osserva, infatti, l'incisione T.T.2 o T.T.3 della Venere Sperimentale create sempre con il bulino ma utilizzando come base di appoggio il blocco di arenaria, si nota che le strie sono meno profonde ma identiche a T.T.1 per morfologia e dimensione. Per cui, si può dire che l'incisione T.T.3 della Venere Grande è stata creata con un bulino ma l'assenza o la presenza di una base di appoggio è difficilmente valutabile. Le aree T.T.2 e T.T.1 della Venere Archeologica purtroppo non sono visibili a causa della presenza di concrezione massiccia sulla sua superficie. Tuttavia al lato sinistro dell'incisione T.T.2 è presente una stria identificabile probabilmente come un errore di esecuzione e che risulta del tipo corta, profonda con profilo ad "U" (C-P-U). La dimensione ridotta della stria $(\mathrm{C})$ è probabilmente dovuta all'errore di esecuzione e per tal ragione non è stata ulteriormente continuata, per cui si può dedurre che, per tipologia di stria, anche il taglio T.T.2 sia stato effettuato mediando l'uso del bulino. L'incisione T.C. mostra delle linee lunghe che seguono l'andamento del collo e delle piccole strie che invece sono disposte in maniera disordinata. Attraverso un confronto morfologico e visivo si è reputato opportuno rapportare questi due tipi di strie all'esperimento 3B2. In entrambe i casi si hanno le tipologie lughe, profonde e con profilo a "U" (L-P-U) e corte, poco profonde, profilo a "V" (C-PP-V) in cui le prime risultano poco coprenti e ordinate e le seconde risultano coprenti e disordinate. Sul Campione 3B2 era stata prima raschiata la superficie, con un raschiatoio immanicato, e poi eseguita un incisione. Si può ipotizzare, quindi, che vi è stato sulla Venere Grande un'azione dello stesso tipo.

Per quanto riguarda la zona del busto: L'area B1 della Venere Archeologica mostra strie uguali per tipologia alla zona B1 della Venere Sperimentale (L-PP-V>C-PP$\mathrm{V})$. L'area sperimentalmente era stata eseguita attraverso l'alternanza del blocco di arenaria e del frammento di arenaria e del solo uso dell'azione abrasiva. La distribuzione disordinata delle strie della Venere Archeologica e il loro orientamento fanno presupporre un'azione bidirezionale dall'alto verso il basso (A-B/B-A) contraria quindi a quella bidirezionale da sinistra a destra (S-D/D-S) della Venere Sperimentale. La diversa azione gestuale è probabilmente dovuta all'area T.C citata precedentemente. In pratica mentre per la Venere Archeologica la parte del collo e quella immediatamente successiva sono state raschiate ottenendo l'abbassamento di superficie, l'abrasione è stata applicata solo per modellare la sinuosità del seno. Segue che il movimento utile per tal scopo è quello che va dalla base del collo alla zona sopra il seno. Nella Venere Campione la parte immediatamente successiva al collo è stata unicamente abrasa e non si è modellata la zona superiore al seno. Le tracce di incisione B.S sono visibili sono nell'attacco del seno destro della Venere Archeologica. Le strie sono di tipologia uguale al taglio T.T.3 (ossia lunghe, profonde con morfologia ad "U", L$\mathrm{P}-\mathrm{U})$ realizzate con il solo bulino e si può anche avanzare l'ipotesi di un'assenza della base di appoggio per la realizzazione della Venere Archeologica. Si ricorda, infatti, che l'aiuto di una base di appoggio comporta una minor profondità di stria legato al fatto che il bulino non toccando unicamente di punta provoca una sorta di una sorta di "abrasione" della superficie durante le fasi di azione bidirezionale che si effettuano. L'area B.2 della Venere Archeologica, ossia la zona tra le linee del seno e le incisioni 
Fig. 10: A: Venere Archeologica, ricostruzione del "matrix gestuale" (M. Basile, con il permesso del museo M.A.R.T.A. di Taranto); B: Venere Campione, ricostruzione del "matrix gestuale" (M. Basile).
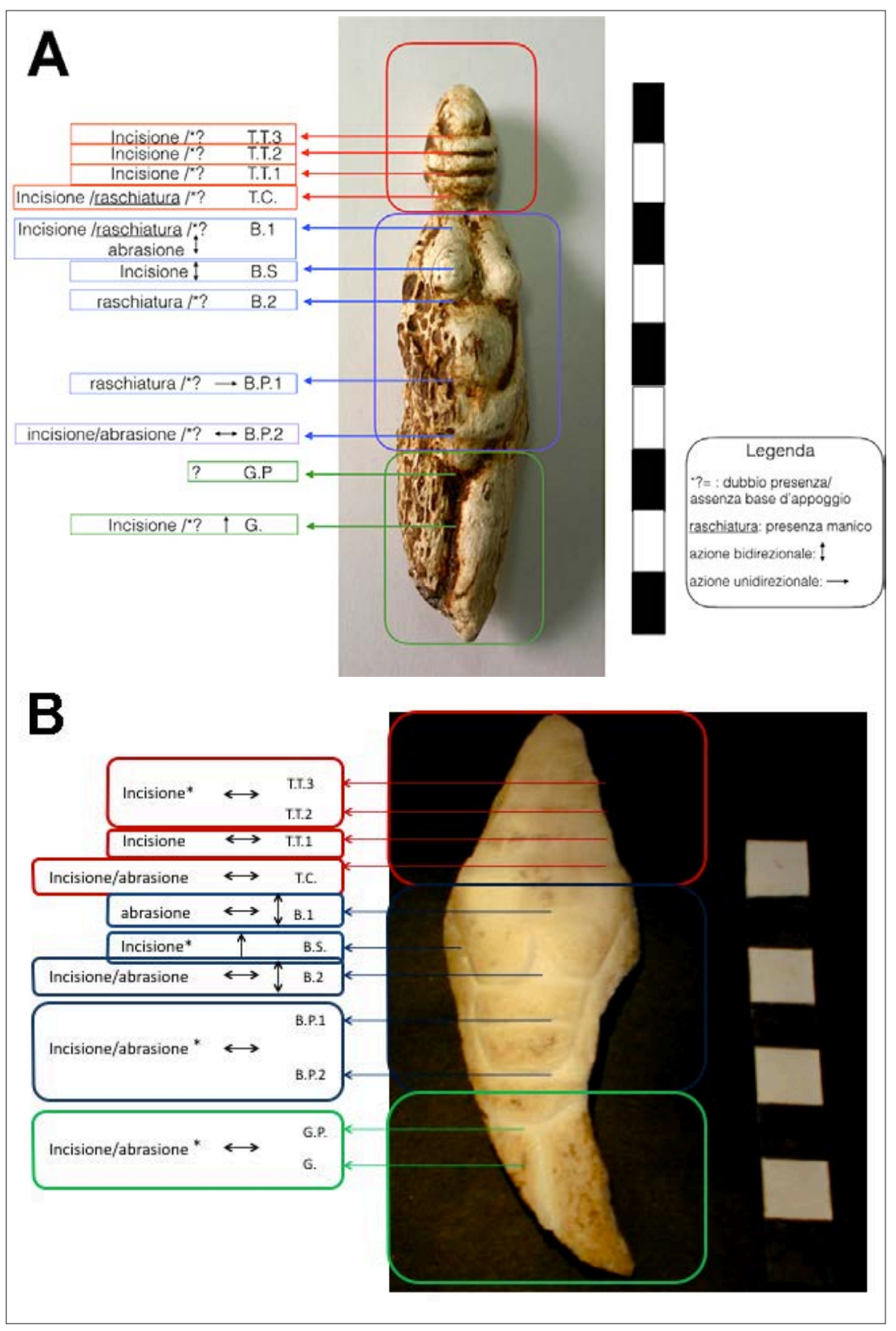

addominali, è stata realizzata, probabilmente, attraverso l'azione della raschiatura con assenza del manico secondo gesto unidirezionale sinistra destra (S-D) visibile nel Campione 3A1. Le strie trovate nella Venere Archeologica hanno morfologia del tipo "solchi a catena" che fanno dedurre una loro realizzazione simile alla sperimentazione. Per quanto riguarda l'area addominale i tagli B.P.1e B.P.2 mostrano diversità di esecuzione nella
Venere Archeologica. La traccia B.P.1 è infatti confrontabile con il Campione 3B1. La traccia d'uso era stata ottenuta mediante raschiatoio immanicato con azione unidirezionale sinistra destra (S-D) producendo strie di morfologia "solco a scalino". Il gesto artistico compiuto quindi per il B.P.1 per uguaglianza di stria potrebbe risultare il medesimo. Per quanto riguarda la traccia B.P.2 la tipologia di stria rientra nell'utilizzo alternato di abrasione 
e incisione attraverso l'uso del bulino e del frammento di arenaria confrontabile quindi con la traccia B.P.2 della Venere Campione. Anche in questo caso l'utilizzo o meno di una base di appoggio per l'esecuzione di abrasione e incisione non è valutabile.

Per quanto riguarda la zona delle gambe: l'area G.P e G. della Venere Archeologica non sono analizzabili a causa dell'enorme quantità di terriccio depositato al suo interno. Tuttavia si è preso in considerazione un taglio presente sulla Gamba sinistra della statuina. Dalle osservazioni al microscopio si è notato che da esso partono delle strie del tipo L-P-U (lunghe, profonde, con morfologia ad "U") che fanno presuppone l'azione dell'incisione. Il taglio era probabilmente già esistente sull'osso o comunque formatosi naturalmente (weathering cracks) ed è stato sfruttato per realizzare la parte terminante della gamba destra della Venere Archeologica, in quanto, parallelo alla naturale conformazione dell'osso che chiude la gamba sinistra.

Il confronto ha dato come esito la realizzazione di un vero e proprio "matrix gestuale" (fig. 10) ossia, un riassunto schematizzato delle azioni effettuate su ogni incisone della Venere Archeologica.

La ricostruzione delle azioni compiute al fine di realizzare la Venere Grande, rende possibile ipotizzare una grande varietà di gesti molto puntuali probabilmente premeditati da una "scaletta di gesti da effettuare". L'alternanza di abrasioni, incisioni e raschiature in determinate aree fa intuire che, l'uomo primitivo, sapeva già cosa voleva ottenere e le procedure da utilizzare per realizzarlo. Il "matrix gestuale" si può cosi sintetizzare:

- La testa della Venere Grande: è stata probabilmente realizzata tramite incisione; il collo invece con alternanza di incisione e raschiatura, con raschiatoio immanicato. In entrambe i casi la presenza o l'assenza di una base di appoggio è dubbia come anche il tipo di azione bidirezionale o unidirezionale.

- Il busto della Venere Grande: la zona concernente la parte iniziale del torace è stata probabilmente raschiata $\mathrm{e}$ incisa, mentre abrasa è la zona al di sopra del seno. Le linee che definiscono il seno sono state effettuate con l'incisone, mentre unicamente raschiate risultano la zona immediatamente sotto il seno e la prima linea della pancia. In entrambe i casi, la linea del seno e la prima linea addominale sono state eseguite con un'azione bidirezionale. L'ultima linea della pancia è stata prodotta tramite alternanza di incisione e abrasione. In tutti i casi è dubbio l'uso di una base di appoggio.
- Le Gambe e il pube: non è stato possibile risalire ad un matrix gestuale a causa di alcune concrezioni presenti in molte fessure della Venere. Nella gamba sinistra è visibile un taglio, probabilmente naturale dell'osso, che è stato lavorato ad incisione in quanto parallelo alla chiusura della gamba destra. L'azione in tal caso è probabilmente unidirezionale.

Si può infine osservare che il "matrix gestuale" della Venere Campione (fig. 10B) risulta limitato alla sola azione abrasiva e dell'incisione e questo suggerisce ancora una volta l'importanza della creazione di una "collezione di confronto" che aumenta il campionario di comparazione.

\section{CONCLUSIONI}

In questa articolo è stato proposto un metodo di ricerca per l'analisi delle Veneri del Paleolitico Superiore sfruttando i principi scientifici dell'archeologia sperimentale. Indagare il mondo dell'arte non è semplice in quanto intriso di simbolismo legato a culture ormai lontane dal nostro presente. Il modo migliore per avvicinarsi e cercare di comprendere alcune di quelle tradizioni e oggetti di cui ormai non si conosce più il significato e il ruolo, è di creare un ponte fra il presente e il passato attraverso l'archeologia sperimentale. L'archeologia sperimentale infatti permette di ricostruire oggetti e gestualità del passato attraverso l'utilizzo di materiali il più possibile affini all'originale e seguendo un protocollo sperimentale. L'obiettivo della ricerca era per l'appunto comprendere la gestualità e le azioni che permettevano la realizzazione delle Veneri paleolitiche e per questo è stato creato un protocollo sperimentale ad hoc. E' stata scelta come Venere Archeologica di riferimento la Venere Grande in osso trovata in località Parabita (Le, Puglia) e datata tra i 22000 e i 20000 B.P.. Il protocollo sperimentale prevedeva:

1. La realizzazione di una "collezione di confronto" utile alla creazione di una serie di tracce tecnologiche effettuate su campioni d'osso, in modo da ottenere un sufficiente numero di elementi da confrontare con le tracce della Venere Archeologica.

2. La realizzazione di una Venere Campione ossia, la produzione sperimentale di una statuina in osso con dettagli anatomici simili alla Venere Archeologica utilizzando repliche di strumenti litici di alcuni materiali rinvenuti nel sito di Parabita. 
3. Infine il confronto tra le tracce tecnologiche sperimentali e quelle archeologiche.

La fase finale della sperimentazione e del confronto ha dato come esito la realizzazione di un "matrix gestuale", vale a dire, un riassunto schematico dei gesti che hanno portato alla realizzazione della Venere Archeologica. Per semplicità la Venere Archeologica e Sperimentale sono state idealmente divise in tre parti: Testa, il Busto e le Gambe. Dai confronti è emerso che la Venere Archeologica è stata realizzata attraverso l'azione combinata di: abrasione, incisione e raschiatura. In particolare si può ipotizzare che le linee della testa sono state realizzate con l'incisione tramite bulino, il collo e l'area che precede il seno, attraverso l'uso di un raschiatoio immanicato alternato ad un gesto di tipo abrasivo con pietra arenaria dura con azione bidirezionale (A-B/B-A). L'area del seno risulta nuovamente incisa tramite bulino con movimento bidirezionale (A-B/B-A), mentre, la zona addominale e la prima linea sulla pancia risultano effettuati tramite l'azione di un raschiatoio non immanicato secondo movenza unidirezionale (S-D). Infine la zona delle gambe non ha dato informazioni circa il modo di realizzazione, tuttavia un taglio naturale dell'osso, situato nella gamba destra della Venere sembra essere stato sfruttato per delineare la linea di chiusura della gamba attraverso l'azione dell'incisione con bulino. A differenza della Venere Archeologica, la Venere Campione è stata realizzata solo attraverso l'uso del bulino e di un frammento e un blocco di arenaria. Per la sua realizzazione si è impiegato un tempo di 638 minuti per un totale di 10 ore di lavoro divisi in 11 giorni di esecuzione. I gesti e le azioni effettuate sono state registrate all'interno di un database così come gli esperimenti condotti per realizzare la "collezione di confronto". L'applicazione di una terminologia semplificata per questo tipo di analisi concernenti il solo utilizzo dei termini "stria" o "solco" non ha in alcun modo agito in senso negativo sul riconoscimento delle varie tracce tecnologiche. In linea generale si può dire che il lavoro sperimentale non è risultato particolarmente complesso in senso artistico e che le azione di incisione, raschiatura e abrasione sono facilmente eseguibili da chiunque. Si può ritenere infatti che le Veneri potevano essere prodotte da uomini, donne e bambini indifferentemente e anche in breve tempo. Se infatti la Venere Campione è stata riprodotta in circa dieci ore di lavoro senza alcuna esperienza iniziale per quanto concerne la lavorazione dell'osso si può immaginare che un individuo del passato, che aveva ormai acquisito metodo e gestualità, poteva impiegare anche solo due ore per realizzare una statuina completa. In conclusione si può dire che, l'applicazione di un metodo di indagine che include procedure sperimentali in un campo così complesso come l'arte può fornire informazioni oggettive circa le modalità che si trovano alla base della loro stessa realizzazione. L'aggiunta a tali informazioni di analisi estetico-tipologiche tendono a completare questo tipo di ricerca rendendola ancora più valida. L'elaborato qui proposto ha tentato di applicare un metodo di analisi sperimentale al campo dell'arte mobiliare paleolitica, creando per tal fine, un protocollo sperimentale e un analisi di tracce tecnologiche ex novo. Il campionario utilizzato per la sperimentazione, seppur limitato dalla disponibilità di materiale conservata all'interno del laboratorio in quel momento, ha fornito come visto fin ora ottimi risultati circa la stesura del "Matrix gestuale". Ulteriori analisi, in tal senso, saranno in seguito utili per alimentare e arricchire questo campo di ricerca.

\section{RINGRAZIAMENTI}

Si ringrazia il Museo di Taranto M.A.R.T.A per l'ospitalità e per aver concesso lo studio della Venere Grande di Parabita . Si ringrazia inoltre il laboratorio LTFAPA, Dipartimento di Scienze dell'Antichità, Università di Roma "Sapienza" e in particolare le professoresse Cristina Lemorini e Daniela Zampetti che hanno permesso la realizzazione di questo lavoro.

\section{BIBLIOGRAFIA}

AVERBOUH, A. (2000): Technologie de la matière osseuse travaillée et implications palethnologiques: l'exemple des chaînes d'exploitation du bois de cervidé chez les Magdaléniens des Pyrénées (Ph.D Thesis), Paris.

BINFORD, R. L. (1968): New Perspectives in Archaeology, Am.J. Phys. Anthropol. 32, 219-220.

CAMPS FABRER, H. (1977): Compte rendu des travaux de la commission de nomenclature sur l'industrie de l'os préhistorique: Méthodologie appliquée à l'industrie de l'os préhistorique, Colloque International sur l'industrie de l'os dans la Préhistoire, Senanque, 19-25.

COLES, J. (1973): Archeologia sperimentale, Milano.

DE MARINIS, R. (2006): L'arte Paleolitica: Dispense del corso di Preistoria, Milano.

D'ERRICO, F.; GIACOBINI, G. (1985): Méthodologique de l'analyse de l'outillage osseux un exemple d'étude, L'Anthropologie 89 (4), 457-472. 
GRIFONI CREMONESI, R. (1987): Due complessi d'arte del paleolitico superiore: la grotta Polesini e la grotta delle Veneri, Atti del VI convegno sulla preistoria-protostoria della Daunia (A. Palma di Cesnola, ed ), San Severo, 35-46.

GRIFONI CREMONESI, R. (1992): Manifestazioni di arte mobiliare dei livelli Epiromanelliani di Grotta delle veneri di Parabita e da grotto Marisa presso Otranto (Lecce), Atti della XXVIII Riunione Scientifica: arte in Italia dal Paleolitico all'età del Bronzo, Firenze.

INGRAVALLO, E. (1997): La passione dell'origine. Giuliano Cremonesi e la ricerca preistorica nel Salento, Catalogo della Mostra, Lecce, Conte, XV, Lecce, 3-16.

PETILLON, J. M. (2006): Des Magdaléniens en armes. Technologie des armatures de projectile en bois de cervidé du Magdalénien Superieur de la Grotte D'Isturitz (Pyrénées- Atlantiques), Artefacts 10, Treignes.

PISCOPO, G.; RAMDILLI, A. M. (1966): Sul rinvenimento di due Veneri Paleolitiche a Parabita, Atti Società Toscana Scienze Naturali LXXIII, 148-156.
PISCOPO, G.; RADMILLI, A. M. (1966): La prima campagna di scavo delle Veneri a Parabita, La Zagaglia: rassegna di scienze, lettere e arti 32, Lecce, 259- 264.

RADMILLI, A. M. (1969): The chronological position of the Venuses of Parabita, Ipek XXII, 35-57.

REYNOLDS, P. J. (1999): The Nature of Experiment in Archeology, Experiment and Design in Archaeology, Studies in honour of John Coles (A.F. Harding, ed), Oxford, 156-162.

RODRIGUEZ, P. (1999): Dio è nato donna. I ruoli sessuali alle origini della rappresentazione divina, Roma.

SARNO, C.(1989): Arte, tempo e simulazione, Napoli.

TUMAZZO, F. (2005): L'archeologia sperimentale: definizione e questioni di metodo, Hortus Musicus XXIII (Ut Orpheus), 70-74.

ZAMPETTI, D.; ALHAIQUE, F. (2004): Aux origines de la représentation: les statuettes paléolithiques de l'Italie centrale et meridionale, Actes du XIVeme Congres UISPP 2001 , Belgio, 187-198. 